\title{
"Double-bundle" tibialis anterior tendon transfer for prevention of pes equinus after Chopart amputation
}

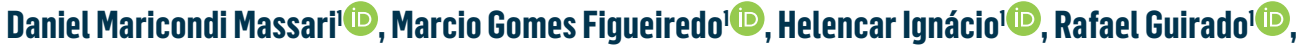 \\ Wilisson Ribeiro Filho' ${ }^{\mathbb{D}}$, José Alexandre Lopes da Silva Alvarenga' ${ }^{(\mathbb{D})}$ \\ 1. Faculdade de Medicina de São José do Rio Preto, São José do Rio Preto, SP, Brazil.
}

\begin{abstract}
Objective: Assess patient performance and quality of the stump after amputation at the Chopart (midtarsal) joint, with double-bundle transfer of the tibialis anterior muscle tendon to the talar neck.

Methods: This study evaluated the medical records of 5 patients who underwent Chopart amputation with double-bundle transfer of the tibialis anterior tendon to the talar neck, assessing pre and postoperative performance and gait.

Results: The patients were operated on between January 2008 and December 2018, and the data obtained from the survey allow us to conclude that, after the proposed procedure, all patients reported an improvement in walking, besides noting a significant reduction in the degree of stump equinus.
\end{abstract}

Conclusion: The surgical technique described in this article produced a significant improvement in patient performance as assessed by the AOFAS hindfoot score, and prevented the formation of ulcers in the anterior region of the stump.

Level of Evidence IV; Therapeutic Study; Case Series.

Keywords: Amputation; Tendon transfer; Equinus deformity; Foot deformities, acquired; Diabetic foot.

\section{Introduction}

Nowadays, due to the exponential increase in auto accidents and greater survival of diabetic patients, amputations of the foot due to high-energy trauma and complications of diabetes mellitus are increasingly present in the lives of orthopedists. Partial foot amputations aim at maintaining the greatest possible length of the lower limb, facilitating and reducing energy expenditure in gait ${ }^{(1)}$. In these amputations, the most proximal level that manages to preserve the height of the lower limb is amputation at the midtarsal (Chopart) joint complex (talonavicular and calcaneocuboid joints) ${ }^{(2,3)}$. For many years, this surgical technique did not have many advocates due to the equinus deformity that forms after this procedure. This equinus is a result of the muscle imbalance caused by the removal of midfoot bones, and consequently, of the tendons responsible for foot/ankle dorsiflexion, such as the tibialis anterior tendon ${ }^{(4)}$. Stretching and/or tenotomy of the calcaneal tendon, combined with transfer of the tibialis anterior tendon to the neck of the talus, are recommended to minimize this complication ${ }^{(1,3,5-8)}$.

The tibialis anterior tendon has a very peculiar characteristic. It features a groove that divides it into two hemitendons, easily identified when we open its sheath. In the past Hoffer et al. ${ }^{(9)}$ advocated the division of the tibialis anterior tendon into two hemitendons, and the transfer of one of these to treat spastic pes varus in cerebral palsy. Other authors have used this transfer to treat residual congenital clubfoot deformities ${ }^{(10,11)}$.

The aim of this work is to assess patient performance and quality of the amputation stump achieved after performing the procedure through the Chopart joint, combined with
Study performed at the Faculdade de Medicina de São José do Rio Preto, São José do Rio Preto, SP, Brazil.

Correspondence: Daniel Maricondi Massari. 225 Manoel José Serpa St., São Carlos, SP, Brazil, Zip Code: 13562-070. E-mail: maricondiortopedia@outlook.com Conflicts of interest: none. Source of funding: none. Date received: October 09, 2019. Date accepted: March 10, 2020. Online: April 30, 2020
How to cite this article: Massari DM, Figueiredo MG, Ignácio H, Guirado R, Ribeiro Filho W, Alvarenga JAL. "Double-bundle" tibialis anterior tendon transfer for prevention of pes equinus after Chopart amputation. J Foot Ankle. 2020;14(1):52-6. 
double-bundle tibialis anterior muscle tendon transfer to the neck of the talus.

\section{Methods}

This study was approved by the Institutional Review Board and registered on the Plataforma Brazil database under CAAE (Ethics Evaluation Submission Certificate) number: 12446219.0.0000.5415.

We performed a review of the medical records of patients operated on between July 1, 2008 and December 31, 2018 and selected 5 patients, all male, with a mean age of 52 years, ranging from 34 to 73 years, who underwent Chopart amputation with double-bundle transfer of the tibialis anterior tendon to the neck of the talus. Prior to the surgical procedure, all patients were clinically evaluated and answered a questionnaire, globally standardized by the American Orthopaedic Foot and Ankle Society (AOFAS) for patient stratification ${ }^{(12)}$, which evaluates criteria of pain, gait, mobility (range of motion), stability and hindfoot alignment (Figure 1). The abovementioned questionnaire was reapplied at the postoperative assessment and a further physical examination was undertaken to evaluate and compare the pre and postoperative data. The preoperative questionnaire consisted of identification details and the patient's medical history, as well as a clinical evaluation of the amputation stump for the presence of ulcers, rating them according to the Wagner classification system, which is a validated system widely used to evaluate the extent of plantar ulcers as well as the presence of infection and/or gangrene.

We started the surgery by performing an Achilles tenotomy. A posterior incision was made in the ankle, about 2 to $4 \mathrm{~cm}$ proximal to the Achilles tendon insertion in the calcaneus. The tendon was isolated and about $2 \mathrm{~cm}$ of this tendon was resected, followed by rigorous hemostasis, mechanical wound irrigation and suturing through subcutaneous layers and skin.

Skin and subcutaneous dorsal and plantar incisions were made on a case-by-case basis. A common characteristic of all cases was the attempt made to preserve as much of the plantar skin and pad as possible to cover the stump (Figure 2), as the plantar skin, due to its special characteristics, is essential for the satisfactory progress of the stump during prosthesis fittings.

The tibialis anterior tendon was identified and disinserted in the medial cuneiform and base of the first metatarsal bone before opening its sheath.

Then the tibialis anterior muscle tendon was split into two hemitendons (Figure 3), in which sutures were applied using 3.0 nylon thread on their stumps (Figure 4). All other tendons were pulled and cut as proximally as possible, so that they included the stump upon retraction.

The surgery continued with the boring of two transosseous talar neck tunnels using $3.5 \mathrm{~mm}$ drills, keeping a safe distance equivalent to the final size of the tunnel orifices.

The tunnels were widened using a small curette. In the next stage, the two hemitendons were passed through the talar tunnels, using an interference thread as a guide, and then su-

\begin{tabular}{|c|}
\hline $\begin{array}{c}\text { AOFAS ANKLE-HINDFOOT SCALE } \\
\text { (100 POINTS TOTAL) }\end{array}$ \\
\hline 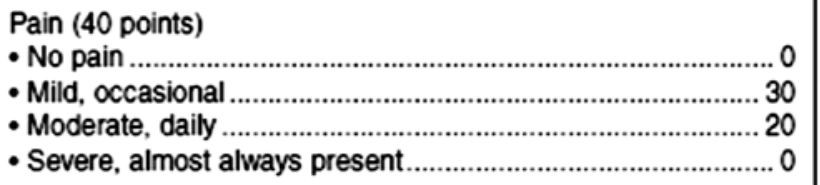 \\
\hline $\begin{array}{l}\text { Functional (50 points) } \\
\text { Restraints in activities, support required } \\
\text { - No restraints, no support.................................................. } 10 \\
\text { - No restraints in daily activities, restrained recreational } \\
\text { activities, no support.................................................... } 7 \\
\text { - Restraints in daily and recreational activities, cane required .. } 4 \\
\text { - Strong restraints in daily and recreational activities; } \\
\text { walker, crutches, wheelchair, orthosis (ankle restraint, } \\
\text { ankle immobilizer)...................................................... } 0\end{array}$ \\
\hline $\begin{array}{l}\text { Maximum walking distance, in blocks } \\
\text { - More than } 6 \\
\text { • } 4 \text { - } 6\end{array}$ \\
\hline $\begin{array}{l}\text { Walking surfaces } \\
\text { - No difficulties in any surface............................................... } 5 \\
\text { - Some difficulty on irregular floors, stairs, steeps and hills ..... } 3 \\
\text { - Strong difficulties on irregular floors, stairs, steeps and hills ... } 0\end{array}$ \\
\hline 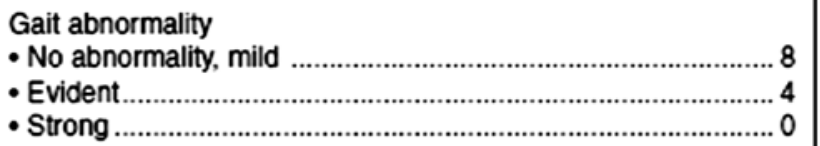 \\
\hline 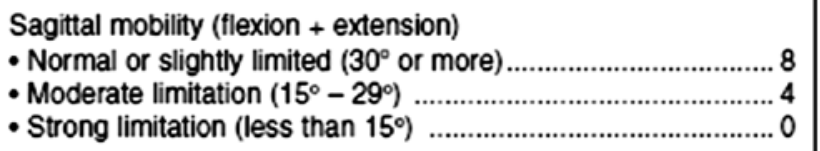 \\
\hline $\begin{array}{l}\text { Hindfoot mobility (inversion }+ \text { eversion) } \\
\text { - Normal or slightly limited }(75-100 \% \text { of the normal mobility)... } 6 \\
\text { - Moderate limitation }(25-74 \% \text { of the normal) ........................ } 3 \\
\text { - Strong limitation (less than } 25 \% \text { of the normal) ........................... } 0\end{array}$ \\
\hline $\begin{array}{l}\text { Ankle-Hindfoot stability (anteroposterior, varus-valgus) } \\
\text { Stable }\end{array}$ \\
\hline 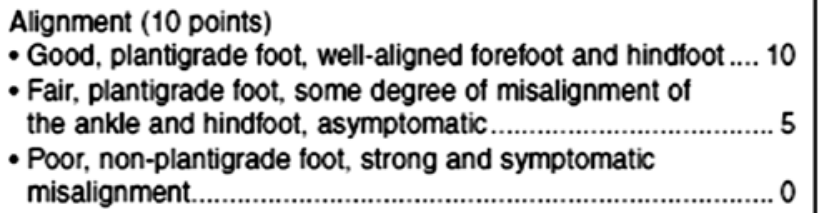 \\
\hline TOTAL \\
\hline
\end{tabular}

Figure 1. AOFAS Scale.

tured together at the bottom of the talus, characterizing their double-bundle reinsertion (Figure 5). 


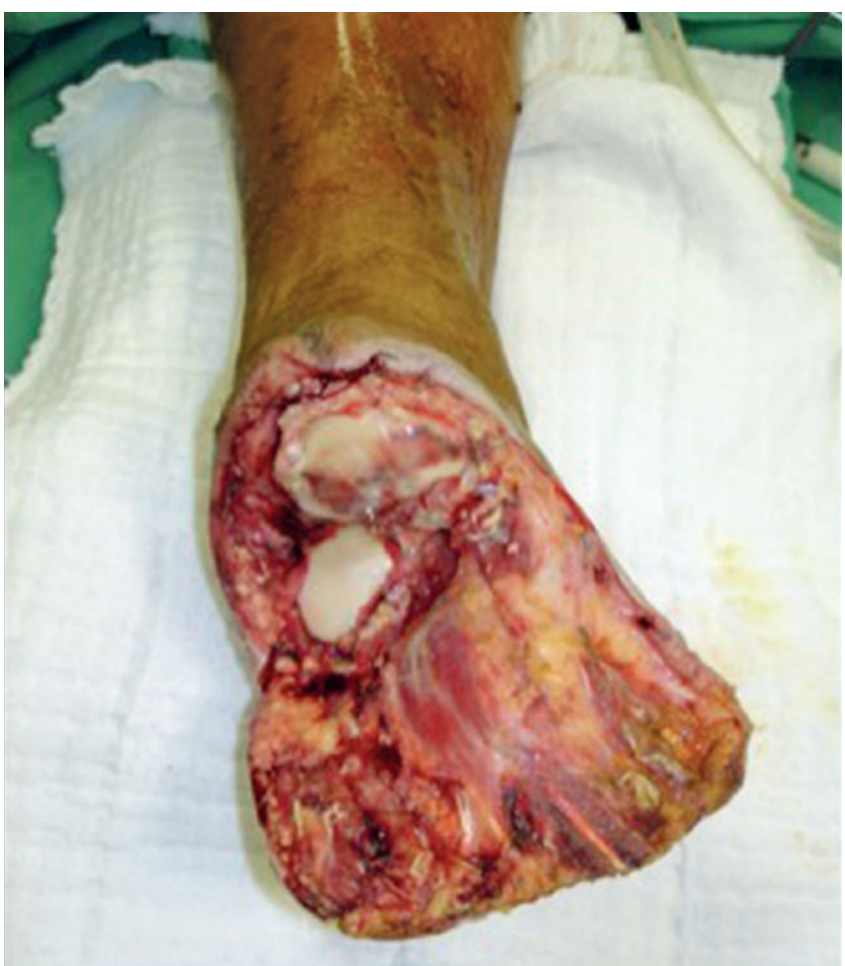

Figure 2. Creation of the flap.

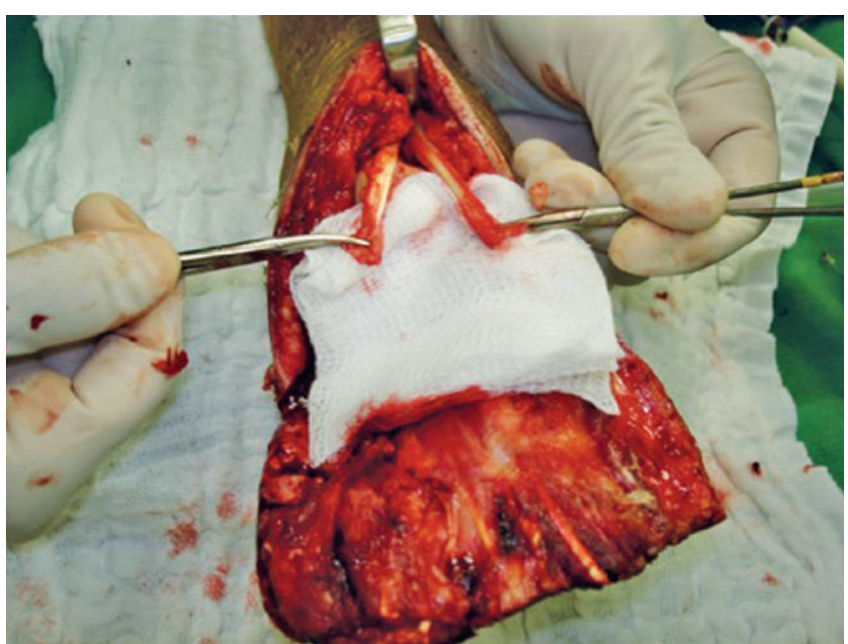

Figure 3. Division of the TA.

We took care to position the stump in slight 5 degree dorsiflexion at this stage of the procedure. After suturing the distal stumps, we sutured the stumps in the area above the talar neck.

Having completed this step, we irrigated the surgical wound, performed hemostasis, inserted a suction drain and closed the stump, once again remembering to leave as much plantar skin from the anterior part of the stump as possible.

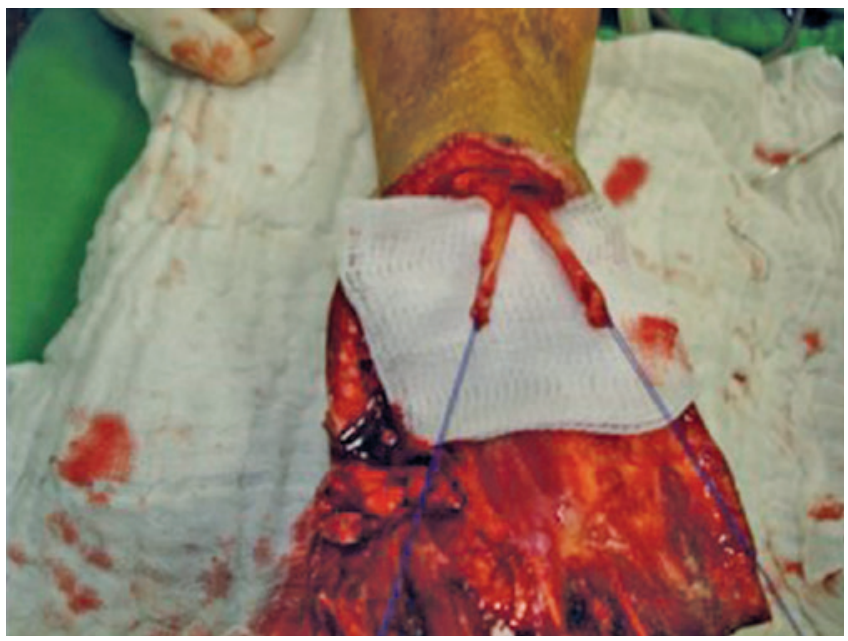

Figure 4. Suturing of the stumps.

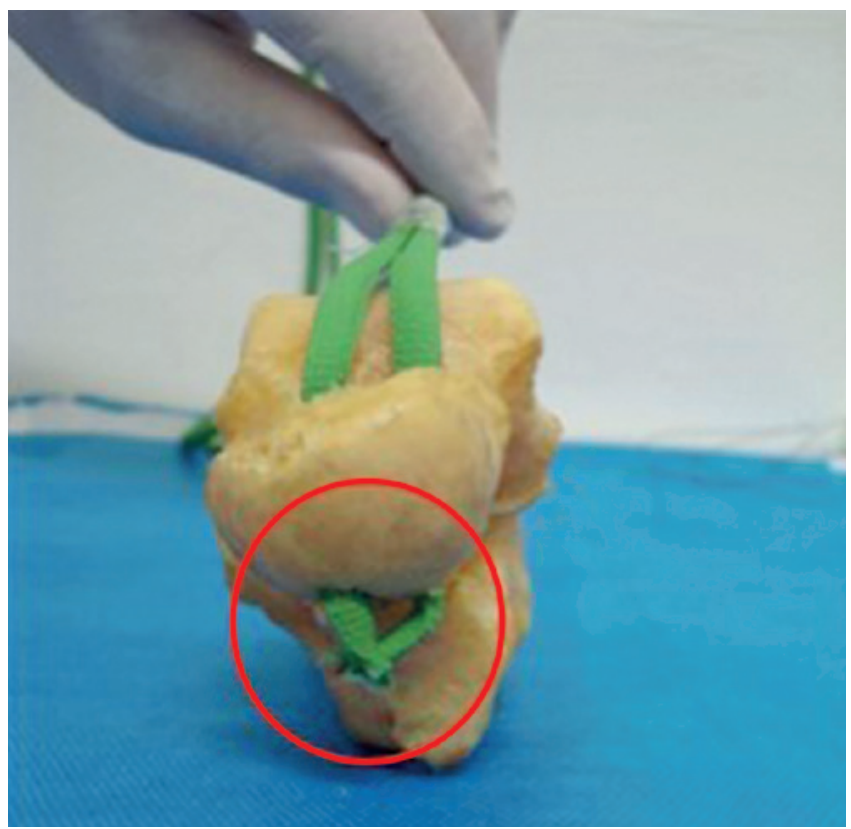

Figure 5. Suturing of the stumps below the neck.

During the postoperative period, the patient wore a plaster splint at 5 degree dorsiflexion continuously for 6 weeks, with no weightbearing during this period. The time to removal of stitches ranged from 3 to 5 weeks. After 6 weeks, if the wound was already properly healed, physiotherapy rehabilitation began, starting gait training and already aiming at future prosthesis fitting ${ }^{(13)}$. Stump ulcers were treated with serial debridement, performed weekly with the application of dressings as directed by the wound dressing group of our institution. 


\section{Results}

A total of 4 patients underwent the final assessment with the AOFAS method, since complications arose in 1 of the patients (dehiscence and infection), leading to the need for further surgical reinterventions, and culminating in a final level of transtibial amputation. Hence this particular patient could not be assessed by the score.

All patients had ulcers in the anterior region of the amputation stump prior to the procedure, while none of them experienced a further episode of ulceration after the procedure. The mean degree of equinus of the stumps was 16.3 degrees preoperatively and 7.3 degrees postoperatively.

In the performance assessment based on the preoperative AOFAS score, the minimum value obtained was 28 points and the maximum was 36 points, with a mean score of 33 points. In the postoperative assessment, the minimum value obtained was 62 points and the maximum was 73 points, with a mean score of 68 points.

Procedural complications included the abovementioned patient where the amputation had to be performed at a higher level, as well as 2 patients who had a small area of skin necrosis near the surgical incision. The latter had a good response to the treatment used by the wound dressing group.

After the double-bundle transfer, all patients reported an improvement when walking. All patients walked at home without needing to use a prosthetic device; 3 used a prosthetic device to walk outside the home and 1 did not even use a device to walk long distances. In the latter case, the patient reports not having adapted to the prostheses he tried to use. All patients were satisfied with the result achieved by the surgery and would have it done again if necessary.

\section{Discussion}

Defining the amputation level is not an easy task for the surgeon ${ }^{(14)}$. We know that the higher the amputation level, the greater the energy expenditure when walking, therefore every effort should be made to maintain the greatest possible limb length and thereby alter gait biomechanics only slightly, with consequent energy savings ${ }^{(1,2,14)}$. Another difficulty frequently observed in amputations at lower limb level is stump healing issues, which can affect up to half of all patients ${ }^{(15)}$.

Among partial amputations of the foot, with the exception of fingers and rays, those that maintain the height of the limb are transmetatarsal procedures and Lisfranc and Chopart amputations. Boyd, Pirogoff and Syme amputations end up shortening the $\operatorname{limb}^{(3)}$.

Transmetatarsal and Lisfranc amputations provide a better functional result due to the preservation of the muscle insertion of ankle flexors and extensors ${ }^{(14)}$. When these two more distal amputations cannot be undertaken, either due to excessive loss of soft tissue or infection, we can use Chopart's amputation.

One factor that caused Chopart's amputation to be relegated to the background for several years was equinus resulting from the muscle imbalance produced by the removal of midfoot muscle insertion points ${ }^{(4,5)}$. To minimize this complication, the stump must have its muscles rebalanced, perfor- ming stretching and/or tenotomy of the calcaneal tendon, combined with the transfer of another tendon to the talar neck in order to function as a dorsiflexor. Surgeons can use the fibularis brevis(3), extensor digitorum, or tibialis anterior tendon ${ }^{(1,3,5-7)}$, with greater preference shown for the latter in the literature. Although our initial sample is small, the results are favorable since we were able to reduce residual stump equinus by more than $50 \%$, a fact that is noteworthy as the act of reducing areas of plantar hyperpressure is one of the pillars of ulcer management, reducing their appearance and recurrence ${ }^{(16)}$. These figures encourage further studies to better assess and publicize the technique.

Some authors maintain that Chopart's amputation is a good alternative in nondiabetic patients who have sustained foot injuries, but they do not indicate the procedure for diabetic patients with Wagner grade 3 ulcers, mainly because the focus of infection may be very close to the foot pad, for which reason it cannot be kept intact. In diabetic patients with active infection (Wagner grade 3), in which there is viability of the foot pad, we recommend performing the procedure in two stages, keeping the surface of the stump open and moist with solid petroleum jelly or vaseline gauze, in the initial stage and after 48 to 72 hours, with improvement of the clinical outlook while maintaining adequate glycemic control, followed by further debridement of devitalized tissues, double-bundle transfer of the tibialis anterior tendon and closure of the stump.

The advantages of this procedure include the non-use of any type of metallic implant, such as screws, washers, interference screws, anchors or metal clamps used to fasten the tendon to the talus; this procedure can be performed when we do not have a sufficient tibialis anterior stump size to pass the tendon through the tunnel and suture the stump at a more proximal point (looping). Suturing between the two hemitendons in the lower part of the talus gives us a greater sense of security than transfer with fixation using transosseous sutures or with support by implants alone, yet we believe that further biomechanical studies are necessary to confirm our preliminary impression. Manufactured bone tunnels have a smaller diameter and by respecting a safe distance in their production, mentioned in surgical technique under the heading of methodology, we reduce the chance of iatrogenic talar neck fractures. In the past, this tunneling technique through the talar neck was believed to predispose patients to talar fractures; however, the occurrence of this type of event was not referred to by Sakaki et al. ${ }^{(8)}$ We can also mention the preservation, albeit slight, of stump dorsiflexion, which considerably improves gait. As we noticed in our results, we were also able to prevent the formation of ulcers in the anterior region of the stump.

Early showed another procedure for the prevention of postChopart's amputation equinus, which consisted of performing tibio-talar-calcaneus arthrodesis fixed with a retrograde nail(1,2). Problems involved in this procedure are the blocking of movement that occurs with arthrodesis and the long time needed for bone fusion ${ }^{(2)}$.

A factor that can be considered negative in this procedure would be the quality of the final part of the tendon, which can result in loosening of stitches between the two hemitendons 
and consequent maintenance of equinus. The clinical data, due to the small number of patients in the sample, are not statistically significant, yet these preliminary results encourage us to continue performing the procedure and, in the future, to gather more sample cases to confirm the effectiveness of this surgical technique.

\section{Conclusion}

The surgical technique described in this article produced a significant improvement in patient performance as assessed by the AOFAS hindfoot score, and prevented the formation of ulcers in the anterior region of the stump.

Authors' contributions: Each author contributed individually and significantly to the development of this article: DMM *(https://orcid.org/O000-00020159-9225) wrote the article, interpreted the results of the study, data collection; HI *(https://orcid.org/0000-0002-1179-4809) conceived and planned the activities that led to the study, approved the final version; MGF *(https://orcid.org/0000-0002-5163-1035) conceived and planned the activities that led to the study, data collection, approved the final version; RG *(https://orcid.org/O000-0001-7878-0711) data collection, statistical analysis; WRF *(https:// orcid.org/0000-0002-2913-8367) data collection, statistical analysis; JALSA *(https://orcid.org/0000-0002-9927-7370) data collection, statistical analysis. *ORCID (Open Researcher and Contributor ID) (iD)

\section{References}

1. Early JS. Transmetatarsal and midfoot amputations. Clin Orthop Relat Res. 1999;(361):85-90.

2. DeGere MW, Grady JF. A modification of Chopart's amputation with ankle and subtalar arthrodesis by using an intramedullary nail. J Foot Ankle Surg. 2005;44(4):281-6.

3. Coughlin MJ, Mann RA, Saltzman CL (editors). Surgery of the foot and ankle. Philadelphia: Mosby; 2007.

4. Millstein SG, McCowan AS, Hunter GA. Traumatic partial foot amputations in adults. A long-term review. J Bone Joint Surg Br. 1988;70(2):251-4

5. Stone PA, Back MR, Armstrong PA, Flaherty SK, Keeling WB, Johnson $\mathrm{BL}$, et al. Midfoot amputations expand limb salvage rates for diabetic foot infections. Ann Vasc Surg. 2005;19(6):805-11.

6. Zgonis $T$ (editor). Surgical reconstruction of the diabetic foot and ankle. Philadelphia: Lippincott, Williams \& Wilkins; 2009.

7. Bowker JH, Pfeifer MA (editors). Levin and O'Neal's the diabetic foot. Philadelphia: Mosby; 2008.

8. Sakaki MH, Sposeto RB, Godoy AL, Ortiz RT, Fernandes TD. Foot amputation at Chopart's joint: surgical technique associated with Achilles tendon stretching and interference screw fixation of anterior tibial tendon transfer. Torbillo Y Pie. 2014;6(1):48-51.

9. Hoffer MM, Reiswig JA, Garrett AM, Perry J. The split anterior tibial tendon transfer in the treatment of spastic varus hindfoot of childhood. Orthop Clin North Am. 1974;5(1):31-8.

10. Kuo KN, Hennigan SP, Hastings ME. Anterior tibial tendon transfer in residual dynamic clubfoot deformity. J Pediatr Orthop. 2001;21(1):35-41.

11. Hui JH, Goh JC, Lee EH. Biomechanical study of tibialis anterior tendon transfer. Clin Orthop Relat Res. 1998;(349):249-55.

12. Rodrigues RC, Masiero D, Mizusaki JM, Imoto AM, Peccin MS, Cohen $\mathrm{M}$, et al. Translation, cultural adaptation and validity of the American Orthopaedic Foot and ANKLE society (AOFAS) anklehindfoot scale. Acta Ortop Bras. 2008;16(2):107-11.

13. Smith DG, Michael JW, Bowker JH (editors). Atlas of amputations and limb deficiencies: surgical, prosthetic and rehabilitation principles. Rosemont: American Academy of Orthopaedic Surgeons; 2004.

14. Chang BB, Bock DE, Jacobs RL, Darling RC 3rd, Leather RP, Shah DM. Increased limb salvage by the use of unconventional foot amputations. J Vasc Surg. 1994;19(2):341-88.

15. Baumgartner R. Forefoot and midfoot amputations. Oper Orthop Traumatol. 2011;23(4):254-64.

16. Armstrong DG, Boulton AJM, Bus SA. Diabetic foot ulcers and their recurrence. N Engl J Med. 2017;376(24):2367-75. 